Hydroécol. Appl. (1994) Tome 6 Vol. 1-2, pp. 59-85

\title{
Bilan des dépôts sédimentaires dans le réservoir de Pareloup (Aveyron, France)
}

\author{
Sediment Budgets in the Pareloup reservoir \\ (Aveyron, France)
}

\author{
Joseph Dagnac \\ Laboratoire d'Hydrobiologie, CNRS URA 695, Université Paul Sabatier, \\ 118, route de Narbonne, 31062 TOULOUSE Cedex, France
}

\begin{abstract}
Résumé. - Pendant près de quarante ans les sédiments se sont accumulés sur le fond du réservoir de Pareloup. Plusieurs essais de caractérisation de ces derniers ont eu lieu depuis 1983, de même que des tentatives d'évaluation du taux de sédimentation par utilisation de pièges à sédiments et analyse de carottes de dépôts.

Il est beaucoup plus aisé et plus fiable d'étudier, lorsque les circonstances le permettent, le sédiment in situ lors d'une vidange. Ce fut le cas à Pareloup pendant l'été 1993. Nous avons donc pu comparer les résultats de l'étude des prélevements in situ avec ceux obtenus antérieurement. La méthode des pièges à sédiments appliquée à ce plan d'eau a donné des résultats assez satisfaisants compte tenu du contexte environnemental. Elle conduisait à proposer un rythme d'accumulation de $1794 \mathrm{t} \cdot \mathrm{km}^{-2} \cdot \mathrm{an}^{-1}$ tandis que l'estimation à partir de la mesure de l'épaisseur réelle des dépôts exondés et du calcul de la superficie qu'ils occupent, avec l'aide de la photographie aérienne, donne un rythme de $3429 \mathrm{t} \cdot \mathrm{km}^{\text {? }}$. an '. Ceci représente une épaisseur de $5 \mathrm{~mm}$ de dépôts secs par an. Leurs caractéristiques physico-chimiques sont proches de celles décrites dans les milieux oligotrophes.
\end{abstract}

Mots-clés. - Sédiment, production, rythmes, granulométrie, caractéristiques chimiques.

Abstract. For nearly 40 years, sediments have been accumulating on the bottom of Pareloup resenoir.

Since 1983, several attempts have been made to characterize them and to estimate the rate of sedimentation by means of sediment traps and analysis of sediment cores.

It is far easier and more reliable, whenever possible, to study sediments "in situ" during draining of a reservoir. Such was the case at Pareloup in summer 1993. We were thus able to compare the results of our study of field samples with those previously obtained. The use of sediment traps in this reservoir gave quite satisfactory results considering the environmental context. They led us to propose a rhythm of accumulation of $1794{\mathrm{t} . \mathrm{km}^{2}}^{2}$.year ${ }^{-1}$, while the estimation on the basis of measurement of the real thickness of deposits after exundation and of calculation of the surface area they occupy (using aerial photography)

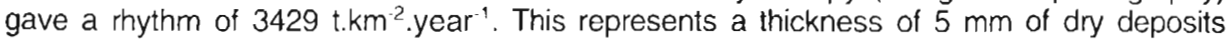
per year. Their physicochemical characteristic are similar to those described for oligotrophic environments.

Key words: - Sediment, yield, rhythms, granulometry, chemical properties. 


\section{INTRODUCTION}

Depuis 1983, l'étude écologique de la retenue de Pareloup a permis au Laboratoire d'Hydrobiologie de l'Université de Toulouse III de collecter une somme importante de données, ce qui l'a conduit à proposer dès 1988, en collaboration avec le département Environnement de l'EDF à Chatou, un modèle de fonctionnement de cet écosystème. Ce modèle a été complété et amélioré en 1993 par J.M. Thebault et M.J. Salençon [19]. Une meilleure connaissance des taux de sédimentation des matières en suspension et une estimation la plus fiable possible du volume de sédiment déposé pouvait permettre d'affiner le modèle pour le compartiment relations eau-sédiments. Profitant de la vidange du réservoir, pendant l'été 1993, nous avons pu compléter les prélèvements et les observations et proposer une synthèse des résultats des différentes campagnes antérieures. Nous avons tenté parallèlement une cartographie des sédiments avec l'appui de photographies aériennes. Trois missions sont actuellement disponibles :

- une mission NB au 1/9000;

- une mission IRC au 1/20000;

- une mission couleur, échelle moyenne: $1 / 6250$.

Les deux premières sont des photographies verticales réalisées le 08.07.93 par la société SPHAIR de Toulouse, la troisième, héliportée, comprend une couverture partielle du site, par bandes de vues subverticales, réalisée par D. Guillemyn ${ }^{(1)}$ le
05.07.93 à bord d'un Ecureuil de la compagnie RODAIR de Rodez. La cartographie des sédiments est en cours d'exécution. De nombreux auteurs anglo-saxons ont traité du problème des dépôts lacustres et ont souligné les limites des différentes techniques d'estimation de leur volume. La vidange d'une retenue est une occasion très particulière qui facilite au maximum le travail d'observation et rend moins aléatoire le prélèvement d'échantillons, elle permet une évaluation plus sûre de la charge en sédiments et une localisation plus précise de la répartition des dépôts de différente nature. Cette répartition géographique des dépôts une fois cartographiée, peut, à posteriori, servir à l'étalonnage de techniques diverses d'observation à distance de type sonar ou séismique réflexion. Les processus qui déterminent le dépôt des sédiments dans les lacs et retenues sont aujourd'hui bien connus (érosion, mise en suspension, sédimentation, consolidation) mais doivent être précisés pour chaque lac en fonction de la morphologie, de l'étendue, de la situation géographique et géologique de son bassin versant.

Les éléments essentiels du cycle biogéochimique qui sont déposés sur le fond d'un plan d'eau sont composés de matériel détritique organique et minéral.

Leur origine peut être allochtone ou autochtone. Dans le premier cas, ces

(1) D. Guillemyn, CNRS UMR 9964, Université Paul Sabatier, 39, allées Jules Guesde, Toulouse. 

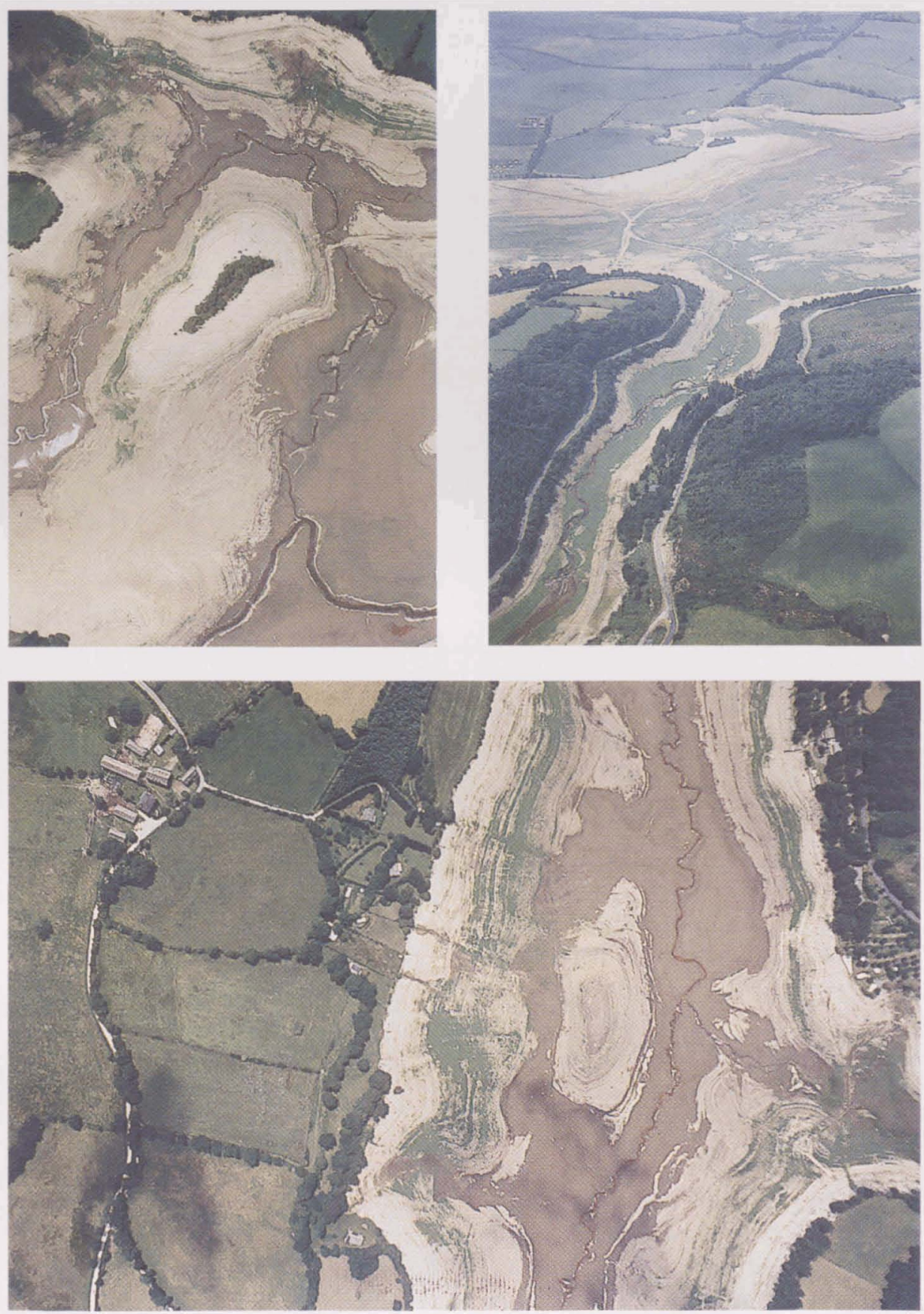

Planche I. - Vues aériennes prises d'hélicoptère en juillet 1993

Plate I. - Aerial views taken by helicopter in July 1993.

Photo 1. - Ile de la côte 805 au nord de Routaboul avec le confluent du Rieutord et du Vioulou. Photo 1. - Island formed at water level 805 north of Routaboul, with the confluence of the Rieutord and the Vioulou.

Photo 2. - Débouché du vallon du Connes au nord-ouest de Salles-Curan.

Photo 2. - Opening of the Connes valley northwest of Salles-Curan.

Photo 3. - Haut-fond entre le Courtal et Notre-Dame d'Aurès dans le vallon du Roucan.

Photo 3. - Shoal between Le Courtal and Notre-Dame d'Aures in the Roucan valley. 
éléments sont apportés par les eaux de ruissellement d'origine variée (ruisseaux, sources, nappes) mais aussi, pour une part non négligeable, par la pluie et le vent. Dans le second cas, il viennent essentiellement des restes de la biomasse vivante s'étant développée dans le plan d'eau.

Lorsque ce matériel tombe sur le fond, il est adsorbé puis progressivement retenu par le sédiment lors des différentes phases de la diagenèse. Mais il peut dans certaines conditions être remis en suspension et il est maintenant admis par de nombreux spécialistes (J.K. Marsh et al. in Mac Manus 1993) que les blooms d'algues nuisibles observés dans Loe Pool depuis 1968 sont en relation directe avec la mise en resuspension par le vent des couches de sédiments riches en éléments nutritifs.

Des expériences de traçage avec des particules fluorescentes ont été réalisées dans un certain nombre de lacs pour mettre en évidence l'influence des vents et des mouvements d'eau induits sur la sédimentation; il y aurait une variation spatiale significative pour les particules inférieures à $20 \mu \mathrm{m}$ dans le cas d'expériences conduites par des vents forts de 10 à $15 \mathrm{~m}^{3} \cdot \mathrm{s}^{-1}$. A Pareloup, lors des épisodes de pompage, les mouvements des masses d'eau ont certainement une influence sur les remises en mouvement des matières en suspension et même sur la remise en suspension de la couche superficielle encore non consolidée du sédiment. Les études conduites sur le site en juin 1988 par les départements EAA et LNH d'EDF avec la participation de la SAT du CEA ${ }^{(2)}$ ont montré que l'eau pompée de Bage dans Pareloup, avec un débit constant de $12 \mathrm{~m}^{3} \cdot \mathrm{s}^{-1}$, allait constituer une masse d'eau se déplaçant essentiellement dans l'axe du Vioulou avec une vitesse maximum sur le fond de $180 \mathrm{~m} \cdot \mathrm{h}^{-1}$. Un autre essai par un fort vent de SE dans l'anse du Rieutord a révélé un déplacement en surface d'une masse d'eau marquée de 310 à $370 \mathrm{~m} \cdot \mathrm{h}^{-1}$ en direction du bassin principal.

Ces deux exemples nous permettent de supposer que les mouvements des masses d'eau doivent avoir une influence non négligeable sur la redistribution spatiale des matériaux les plus fins.

\section{SITUATION ET RAPPEL DE QUELQUES CARACTÉRISTIQUES ESSENTIELLES}

\section{I.1 Géographie}

Le plan d'eau de 1260 ha environ et de $120 \mathrm{~km}$ de périmètre se situe dans le département de l'Aveyron, dans les monts du Lévezou, au SE de Rodez.

Le barrage construit à un point d'étranglement de la vallée du Vioulou, affluent du Viaur, retient à la côte maximale $(805 \mathrm{~m})$ environ $169 \mathrm{hm}^{3}$ d'eau. Avec le Vioulou deux autres ruisseaux principaux alimentent le réservoir, ce sont le Connes et le Rieutord.

(2) M.J. Salençon et P. Calmels 1994, Hydroécol. appl., 6(1/2) : 19-58. 
Le bassin versant de Pareloup peut ainsi être subdivise en quatre sousbassins :

- le bassin versant direct $\left(40 \mathrm{~km}^{2}\right)$ que l'on peut limiter à l'embouchure des trois principaux ruisseaux suscités et qui reçoit en plus les eaux de sept petits ruisseaux secondaires à faible débit ;

- les bassins versant du Vioulou $\left(56,2 \mathrm{~km}^{2}\right) \mathrm{du}$ Connes $\left(21 \mathrm{~km}^{2}\right)$ et du Rieutord $\left(15,3 \mathrm{~km}^{2}\right)$.

La moyenne annuelle des précipitations $(1100 \mathrm{~mm})$ est élevée et les pluies sont réparties assez régulièrement tout le long de l'année avec cependant des minima correspondant à la saison d'été et des maxima en automne et hiver.

Les débits du Vioulou les plus importants se rencontrent de novembre à février avec des valeurs maximales comprises entre 5 et $10 \mathrm{~m}^{3} \cdot \mathrm{s}^{-1}$.

Les vents parfois violents, de secteur Nord ou Sud, balayent le plateau du Lèvezou et peuvent jouer un rôle important dans le remaniement des particules les plus fines de sédiment, soit par transport direct, soit par effet secondaire en créant des vagues à la surface du plan d'eau et des mouvements de masses d'eau en profondeur.

\section{I.2 Géologie}

Si l'on se rapporte à la carte géologique de Salles-Curan on peut remarquer que les roches qui constituent l'ossature du bassin versant de la retenue sont essentiellement métamorphiques; elles peuvent se rattacher à trois grands types : les granitoïdes, les gneiss et les micaschistes. Le bassin versant du Vioulou, le plus grand, est constitué par des métagranites anatectiques alcalins et des orthogneiss à feldspath potassique. Celui du Connes est encaissé dans des gneiss fins à passées micaschisteuses; ces roches présentent des associations minérales composées essentiellement de quartz $(50 \%)$, de plagioclases, de chlorite et de micas (muscovite et biotite) qu'accompagnent des minéraux moins fréquents comme le grenat, la staurotide ou le disthène. Ce dernier bassin est aussi concerné dans sa partie occidentale par les monzogranites et les syenogranites calco-alcalins à texture porphyroïde. Le bassin du Rieutord, pour l'essentiel, correspond à un substrat de monzogranites identiques à ceux du Connes.

Le bassin versant direct de Pareloup présente des affleurements variés de monzogranites et syénogranites calco-alcalins à texture porphyroïde, de métagranodiorites, de gneiss fins feldspathiques et de métagranodiorites anatectiques (alcalins). Dans la partie NE du plan d'eau affleurent, sur une superficie d'environ $3 \mathrm{~km}^{2}$, des formations secondaires de grès arkosiques et cherts; elles s'étendent au Sud de Canet de Salars dans le secteur du Caussanel, formant des plages de sables quartzeux très grossiers. A cette formation de trias terminal peuvent sans doute être rattachés les affleurements de calcaires dolomitiques bien lités que l'on trouve dans les anciennes carrières exploitées pour le four à chaux, aujourd'hui sous 


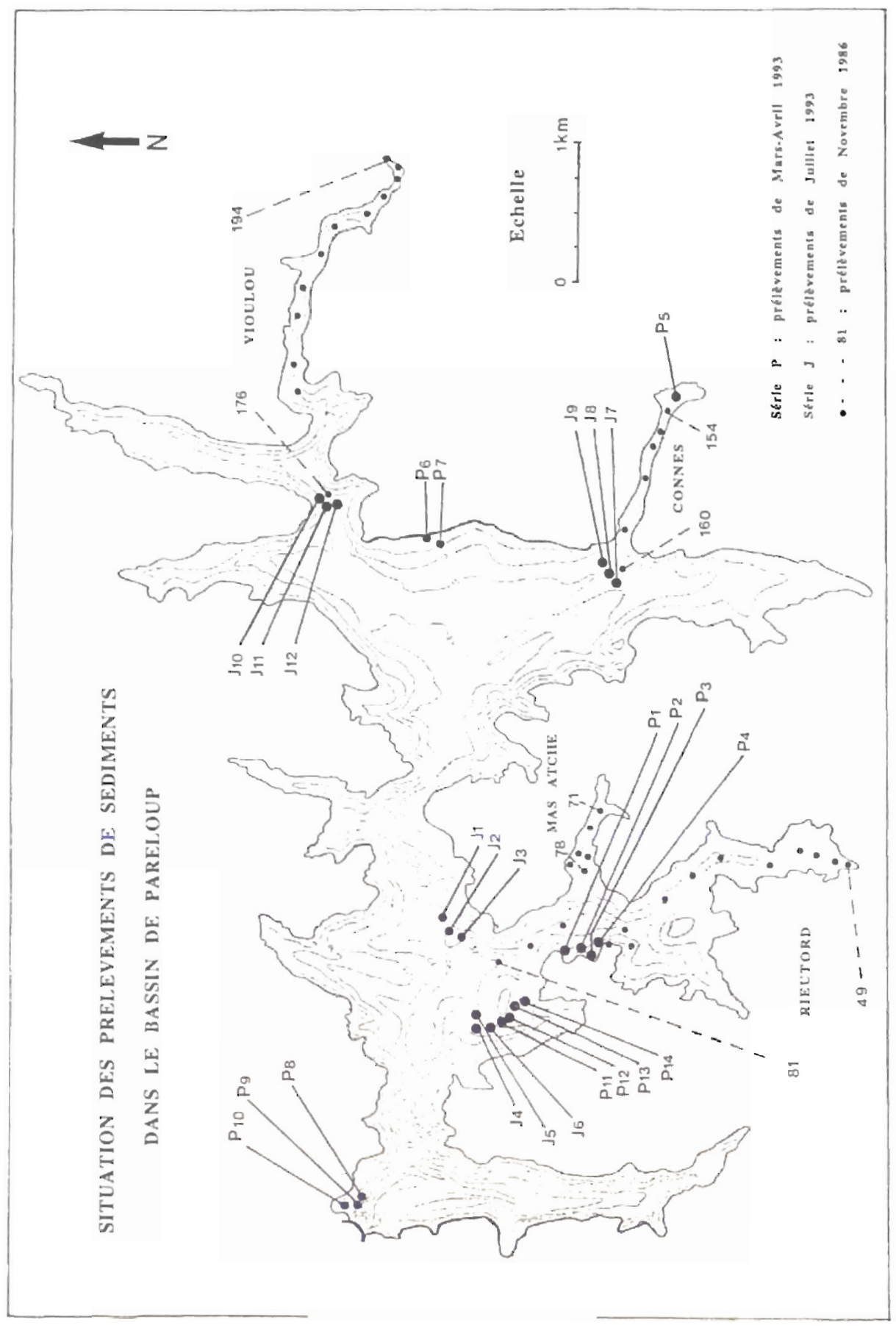


les eaux, sur la rive gauche du Vioulou en amont du pont des 15 arches.

\section{MÉTHODOLOGIE}

\section{II.1 Prélèvement des échantillons} (fig. 1)

Plusieurs essais de caractérisation des sédiments déposés en différents points du plan d'eau ont été réalisés de 1982 à 1993, la retenue étant en eau. Les échantillonnages ont alors été effectués soit par carottage avec un carottier CEMAGREF (Rofes G., 1983) soit à l'aide de la benne Eckmann (Bosc, 1986). Les derniers prélèvements, parfaitement localisés, que nous avons faits en 1993, pendant la vidange et pendant la période d'assèchement du plan d'eau, à la pelle bêche ont été plus aisés à réaliser; ils nous ont permis de récolter des tranches de sédiment consolidés qui nous permettront d'effectuer prochainement une analyse micromorphologique précise.

\section{II.2 Analyses physico-chimiques}

La matière organique et l'azote total ont été dosés sur la même prise d'essai, soit par voie chimique classique (méthodes Anne et Kjeldahl) et par chromatographie en phase gazeuse; nous préférons la méthode classique car dans le cas de sédiments hétérogènes elle permet une prise d'essai plus importante, de l'ordre du gramme, alors que par voie physique la prise d'essai doit être très faible
(10 mg). Les éléments minéraux totaux ont été extraits, après une prédigestion à l'acide nitrique, par chauffage au four à $550^{\circ}$ et reprise à l'acide chlorhydrique et dosés soit par colorimétrie automatisée, soit par absorption atomique. La texture du sédiment, après attaque à l'eau oxygénée, a été déterminée par sédimentation et emploi de la pipette de Robinson ou par l'utilisation d'un granulomètre laser type Cilas Alcatel modèle 715 .

\section{LES APPORTS}

Comme nous l'avons rappelé, la sédimentation dans un réservoir est due à l'accumulation sur le fond de matériel allochtone ou autochtone.

Pour le matériel allochtone les matériels en suspension amenés par les principaux cours d'eau constituent une des composantes les plus évidentes.

La fraction organique qui compose une partie non négligeable des sédiments est elle dérivée, pour l'essentiel, de la précipitation des restes d'organismes vivants (essentiellement zooplancton et phytoplancton) s'étant développés dans la masse d'eau.

Notons que pour cette étude il n'a pas été tenu compte des apports de MES provenant des eaux de pompage de Bage qu'il faudrait à l'avenir prendre en compte. 


\section{III.1 Les apports par les cours d'eau de matières en suspension (inférieures à $0,45 \mu \mathrm{m}$ )}

Pour le plan d'eau étudié, les apports de matière sont surtout dus aux trois principaux ruisseaux qui l'alimentent: le Vioulou, le Rieutord et le Connes.

De ces trois ruisseaux, le Vioulou est celui qui présente les plus forts débits, avec des débits minimum de $0,73 \mathrm{~m}^{-3} \cdot \mathrm{s}^{-1}$ et maximum de 3,53 $\mathrm{m}^{3} \cdot \mathrm{s}^{-1}$ en 1984 (le débit moyen annuel serait de $1,36 \mathrm{~m}^{3} \cdot \mathrm{s}^{-1}$ ).

Les mesures effectuées en 1985 sur le Vioulou lors de l'étude d'une crue ont montré que les quantités de MES augmentent rapidement avec les débits lors de la montée des eaux avec un maximum de $186 \mathrm{mg} \cdot \mathrm{I}^{-1}$ puis diminuent rapidement et fortement et se stabilisent autour de valeurs proches de $20 \mathrm{mg} \cdot \mathrm{I}^{-1}$ malgré des hauteurs d'eau encore élevées. Notons que lors de cette même étude il a été constaté que seul le phosphore total est fortement correlé aux MES ( $r=0,95)$.

Ceci s'explique par le fait que l'effet de chasse d'eau provoqué par la brusque augmentation du débit nettoie le lit du ruisseau emportant les dépôts fins en premier. Les fractions particulaires du phosphore étant majoritaires, le processus physico-chimique de rétention de ce dernier dans les vases s'inverse lors de leur remise en suspension et fait place à un processus de désorption. Le tableau 1 donne les valeurs moyennes des MES des trois ruisseaux; on peut constater que malgré des différences importantes du débit ces valeurs sont assez proches. Pour les années 1984-1985 les débits maximum des trois ruisseaux ont été respectivement de :

$$
\begin{aligned}
& V=6,27 \mathrm{~m}^{3} \cdot \mathrm{s}^{-1}, R=2,07 \mathrm{~m}^{3} \cdot \mathrm{s}^{-1}, \\
& C=1,82 \mathrm{~m}^{3} \cdot \mathrm{s}^{-1} .
\end{aligned}
$$

Les valeurs maximales instantanées de MES enregistrées lors de fortes pluies, pendant la période 1984-1985, sont pour le Rieutord de $274 \mathrm{mg} \cdot \mathrm{I}^{-1}$ (pour $0,89 \mathrm{~m}^{3} \cdot \mathrm{s}^{-1}$ ), pour le Connes de $78,3 \mathrm{mg} \cdot \mathrm{I}^{-1}$ (pour $0,79 \mathrm{~m}^{3} \cdot \mathrm{s}^{-1}$ ) et pour le Vioulou de $117,9 \mathrm{mg} \cdot \mathrm{l}^{-1}$ (pour 2,81 $\mathrm{m}^{3} \cdot \mathrm{s}^{-1}$ ).

Si l'on tient compte des résultats des mesures de 1986, seul suivi permanent à notre disposition sur les trois ruisseaux, c'est près de 1049 tonnes de matières en suspension qui ont été apportées au plan d'eau en un an $(V=714, R=158$, $C=177$ ). En 1987 le Vioulou seul nous donne une valeur de $879,2 \mathrm{t} \cdot \mathrm{an}^{-1}$.

\section{III.2 Taux de sédimentation dans le plan d'eau}

Aux matières en suspension amenées par les ruisseaux s'ajoutent les particules provenant de l'érosion du bassin versant direct du résenoir et surtout des berges, ainsi que la matiere organique provenant de la production de phytoplancton et de zooplancton. Il faudrait aussi tenir compte des particules amenées par les précipitations et les vents. Pour évaluer le taux de sédimentation, des pièges à sédiment ont été mis en place en mars 1985 à décembre 1987; ils étaient constitués 
Tableau I. - Caractéristiques physico-chimiques des trois principaux cours d'eau (notamment les MES).

Table I. - Physicochemical characteristics of the three principal streams in the study (in particular, suspended solids [MES]).

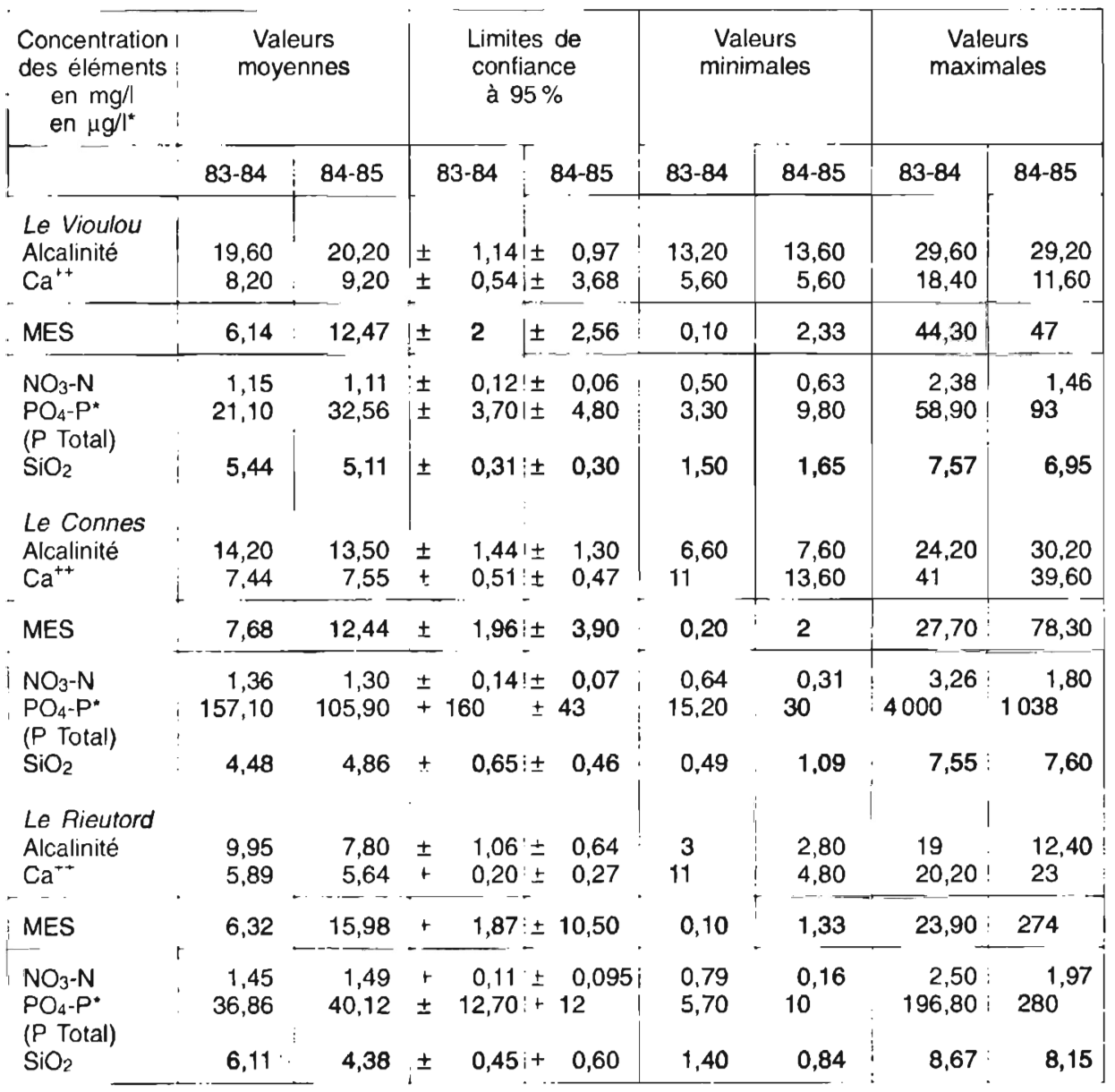

de casiers en PVC contenant chacun 4 bouteilles de $500 \mathrm{ml}$ dont le col était prolongé d'un tube de plastique de $11 \mathrm{~cm}$ de long et de $1,9 \mathrm{~cm}$ de diamètre. Ces casiers, lestés et reliés par un filin à une bouée, étaient immergés à différentes profondeurs de manière à mettre en évidence un éventuel gradient dans la colonne d'eau $(-5,-10,-15,-20,-25$ mètres).

Les résultats n'ayant montré aucune variation significative en fonction de la profondeur, nous avons ici retenu la valeur moyenne entre 10 et 20 mètres de profondeur. 


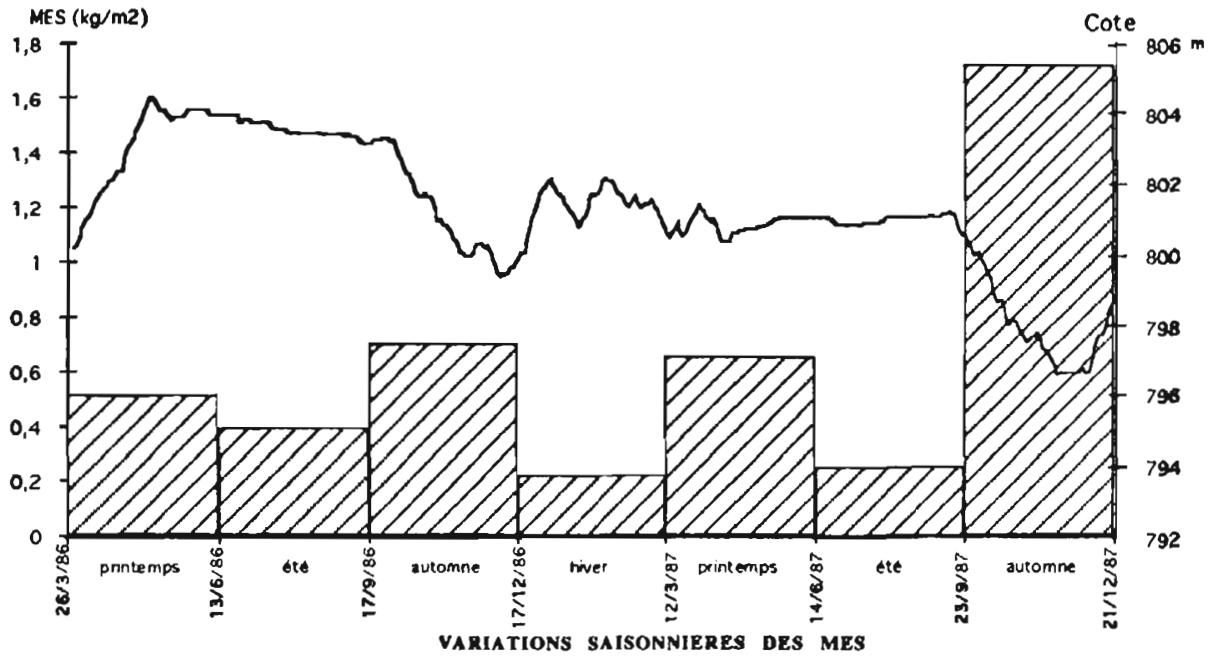

- Les histogrammos représentent les taux mensuels cumulés de MES.

- La courbe indique les variations annuelles de la core du lac.

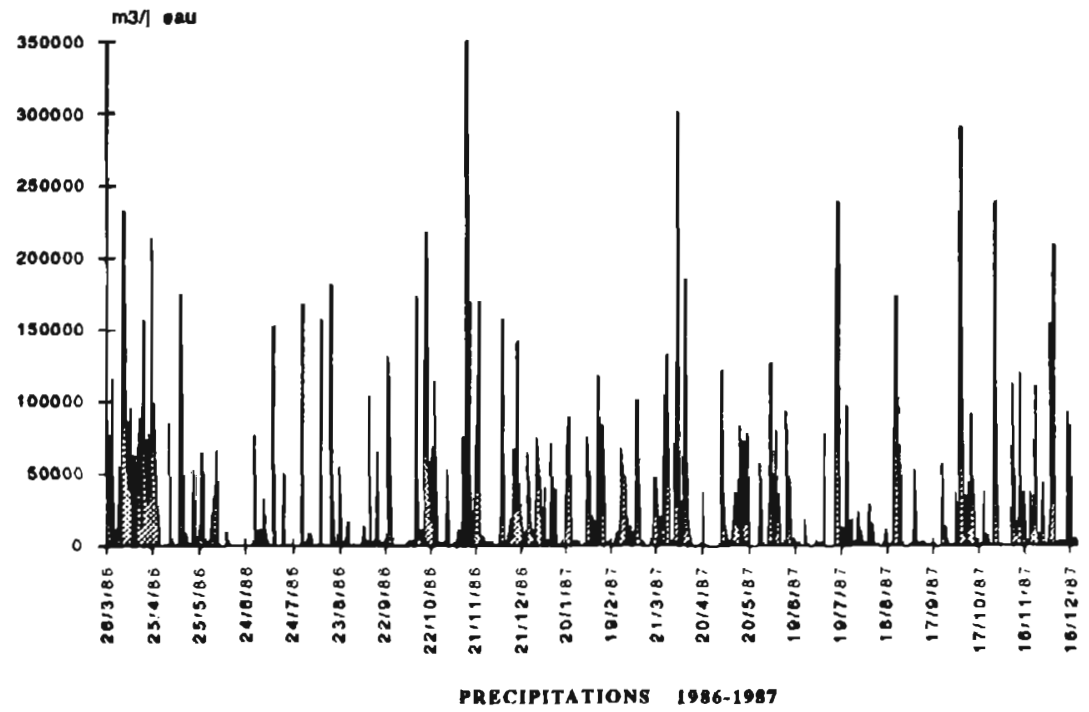

Fig. 2. - Comparaison des précipitations, des variations du niveau d'eau et des MES.

Fig. 2. - Comparison between rainfall, variations in water level and suspended solids (MES). 
La figure 2 résume les principaux résultats obtenus; elle présente deux figures qui, comparées, mettent nettement en évidence quelques corrélations.

Pendant la période de marnage, comme l'indique la courbe de variation de la côte du plan d'eau, à l'automne, l'érosion doit être intense sur les berges et les minima correspondent bien aux taux les plus élevés de matière sèche recueillie dans les pièges. Cette période de l'année correspond également à de fortes pluies.

Mais les taux de sédimentation sont également corrélés, comme le montrent les études précédentes, à la biomasse de phytoplancton telle qu'elle ressort de l'analyse des pigments. L'automne coincide avec la déstratification donc avec la remise en suspension des sédiments et le deuxième maximum des diatomées.

Les diatomées constituent l'essentiel des restes issus de la production primaire que l'on retrouve dans les sédiments.

Comme le signalaient M.J. Salençon et al. [18] pour 1987: "la phase de clarification des eaux observée fin mai résulterait donc plus d'une sédimentation directe des diatomées, consécutive à l'affaiblissement de la turbulence que d'une augmentation du broutage".

Lors de la première tentative de pigeage de mai à août 1985 , pour 93 jours, c'est $340 \mathrm{mg}$ de sédiment qui se déposaient sur $2,8 \mathrm{~cm}^{2}$, soit envi- ron $1215 \mathrm{t} \cdot \mathrm{km}^{-2}$ pour 3 mois en début de saison estivale.

Si l'on se réfère à la période du 26.03.1986 au 14.11.1986 pour 233 jours, $389 \mathrm{mg}$ de sédiment sec correspondent au total des dépôts pour une surface de $2,8 \cdot \mathrm{cm}^{2}$, ce qui représenterait un poids de $180 \mathrm{~g} \cdot \mathrm{m}^{-2} \cdot \mathrm{mois}^{-1}$, soit au maximum, si l'on considère que les 4 mois d'hiver sont généralement moins productifs, $2160 \mathrm{t} \cdot \mathrm{km}^{-2}$. $\mathrm{an}^{-1}$.

Mais les dépôts sont variables tout au long de l'année comme le montre le tableau II pour 1987 et pour une surface de $2,8 \mathrm{~cm}^{2}$; il est donc préférable de calculer l'accumulation sur un cycle annuel complet et en fonction des moyennes saisonnières. Ceci, d'après le tableau II, nous donne pour 1987 un rythme de dépôt de $1794 \mathrm{t}$. $\mathrm{km}^{-2} \cdot \mathrm{an}^{-1}$.

D'après Butcher et al. [4], l'étude de vingt huit réservoirs de la région Pennine en Grande-Bretagne a fait apparaître une grande variation dans l'estimation du rythme de sédimentation; de 0 à $1074,66 \mathrm{t} \cdot \mathrm{km}^{-2} \cdot \mathrm{an}^{-1}$. Les valeurs proposées dans le cadre de notre étude sont plus élevées que celles proposées dans l'étude précédente mais ici la configuration géomorphologique et les paramètres climatiques sont bien différents.

En Cornoudailles le lac de Loe Pool étudié par les chercheurs du PoIytechnic South West, offrant un sédiment de type gyttja brun-rouge, aurait d'après Pickering (in Marsh et al. 1993) un rythme de sédimentation de $100 \mathrm{t} \cdot \mathrm{km}^{-2} \cdot \mathrm{an}^{-1}$. Mais ce lac est 
Tableau II. - Variations saisonnières des dépôts dans les pièges.

Table II. - Seasonal variations in deposits in sediment traps.

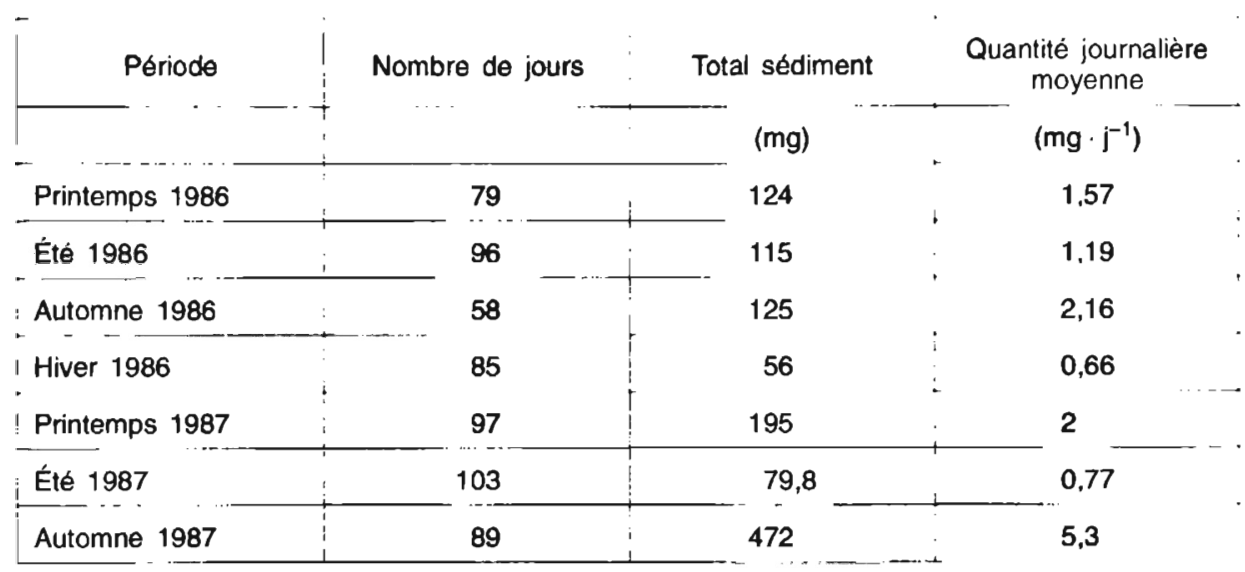

peu profond $(3,6 \mathrm{~m})$, eutrophe et d'un faible volume d'eau $\left(2,1 \cdot 10^{6} \mathrm{~m}^{3}\right)$ comparé à celui de Pareloup (169. $\left.10^{6} \mathrm{~m}^{3}\right)$.

Ces valeurs obtenues par les pièges sont, comme le soulignent de nombreux auteurs, souvent très éloignées de la réalité. II faudrait tenir compte de la géomorphologie du bassin d'accumulation, la pente ayant sans conteste une grande importance sur l'épaisseur et le remaniement des dépôts.

D'après Wetzel [21] les fines particules restent en suspension dans les eaux turbulentes de l'épilimnion et peuvent se déposer sur les marges; mais lors de la destratification elles peuvent être remises en suspension et se déposer alors sur toute la surface du lac. Si l'on ajoute à cela l'action des pluies et des vents sur la zone découverte par le marnage, on peut se douter que les particules minérales ou organiques peuvent avoir un cheminement assez long avant d'être définitivement piégées dans le sédiment.

Les rares épisodes de poussières éoliennes sahariennes qui atteignent l'hexagone au moins une ou deux fois par an, d'après Bucher et Lucas [3] peuvent parfois amener des dépôts non négligeables. Si on se réfère à la pluie de boue de juillet 1983 qui a partout déposé dans le Sud de la France au moins $1 \mathrm{~g}$ par mètre carré (jusqu'à $6 \mathrm{~g}$ dans les Hautes Pyrénées) ce serait un minimum de 1 tonne de poussières qui aurait directement alimenté le plan d'eau de Pareloup. Ceci représenterait 1/2000 du dépôt annuel et donc ici n'aurait qu'un très faible impact.

\section{III.3 Les sédiments exondés}

Plusieurs essais de caractérisation des sédiments déposés en différents 
points du réservoir ont été réalisés depuis 1982 (Rotes G. en 1983, Rhodes T.E. et Le Cohu R. en 1987) soit en prélevant les échantillons à l'aide de la benne Eckmann ou de carottiers. Nous avons analysé des échantillons prélevés à l'aide de la benne Eckmann en 1987 par J.-N. Tourenq et surtout prélevé in situ à la bêche les dépôts au fur et à mesure de la baisse du niveau d'eau qui s'est étalée sur toute la saison du printemps 1993. Début juillet 1993, la vidange de Pareloup étant terminée, nous avons pu, in situ, prélever des portions complètes sur toute l'épaisseur des dépôts (maximum $20 \mathrm{~cm}$ ) les sédiments ayant commencé à sécher en place.

Ceux-ci reposaient dans la plaine centrale sur d'anciens sols de prairies qui bordaient le Vioulou.

Cette facilité de prélèvement nous a permis de multiplier les échantillons et d'en réserver un certain nombre pour une étude micromorphologique ultérieure. Ces prélèvements et les observations qui suivent ont été réalisés entre le 15 et le 18 juillet 1993. Les dépôts mis à l'air ont été soumis à un ressuiement progressif, en fonction de leur épaisseur et de la morphologie du terrain. En séchant la "vase" se fracturait en nombreux polygones irréguliers de quatre à cinq côtés, bien individualisés, séparés par des fentes de un à plusieurs centimètres (photo $n^{\circ} 4$ ). Ce phénomène étant directement lié à la teneur en eau du sédiment, les surfaces encore très humides (Teh $=73$ à 100\% d'eau) n'étant pas craquelées tandis que les fentes apparaissaient à partir de $50 \%$ de teneur en eau environ et que les polygones les plus secs et bien individualisés présentaient une teneur en eau voisine de $40 \%$ par rapport au poids sec; la surface des polygones semblant d'ailleurs liée à l'épaisseur du sédiment. Les petits polygones de $5 \mathrm{~cm}$ d'épaisseur présentant une diagonale moyenne de $15 \mathrm{~cm}$, alors que les plus grands pour une diagonale de $90 \mathrm{~cm}$ correspondaient à l'épaisseur maximale du sédiment soit $20 \mathrm{~cm}$. Après avoir mesuré sur 10 transects de $50 \mathrm{~m}$ à intervalles de 5 mètres l'épaisseur des dépôts, nous avons estimé à $15 \mathrm{~cm}$ l'épaisseur moyenne du sédiment sec dans la plaine principale. Ces dépôts de couleur variable suivant leur état d'oxydation et d'humidité montrent une succession de lits fins millimétriques alternativement clairs et sombres. A l'état humide (Teh : 60 à $80 \%$ ) ils sont de couleur gris très sombre (10 YR $3 / 1)^{(3)}$ ou brun-gris très sombre (10 YR 3/2), tandis qu'à l'état sec (Tech : moins de $40 \%$ ) la couleur varie du gris (10 YR 6/1) au brun (10 YR 5/3).

Leur texture homogène et leur teneur en eau encore assez importante, du 5 au 8 juillet 1993, lors des différentes missions photographiques aériennes, permettront de réaliser une cartographie précise de leur étendue. Une première interprétation de la mission IRC au $1 / 20000$ nous a permis d'évaluer la surface couverte par les dépôts fins occupant le fond des val-

(3) D'après la charte des couleurs de sol de Munsell. 

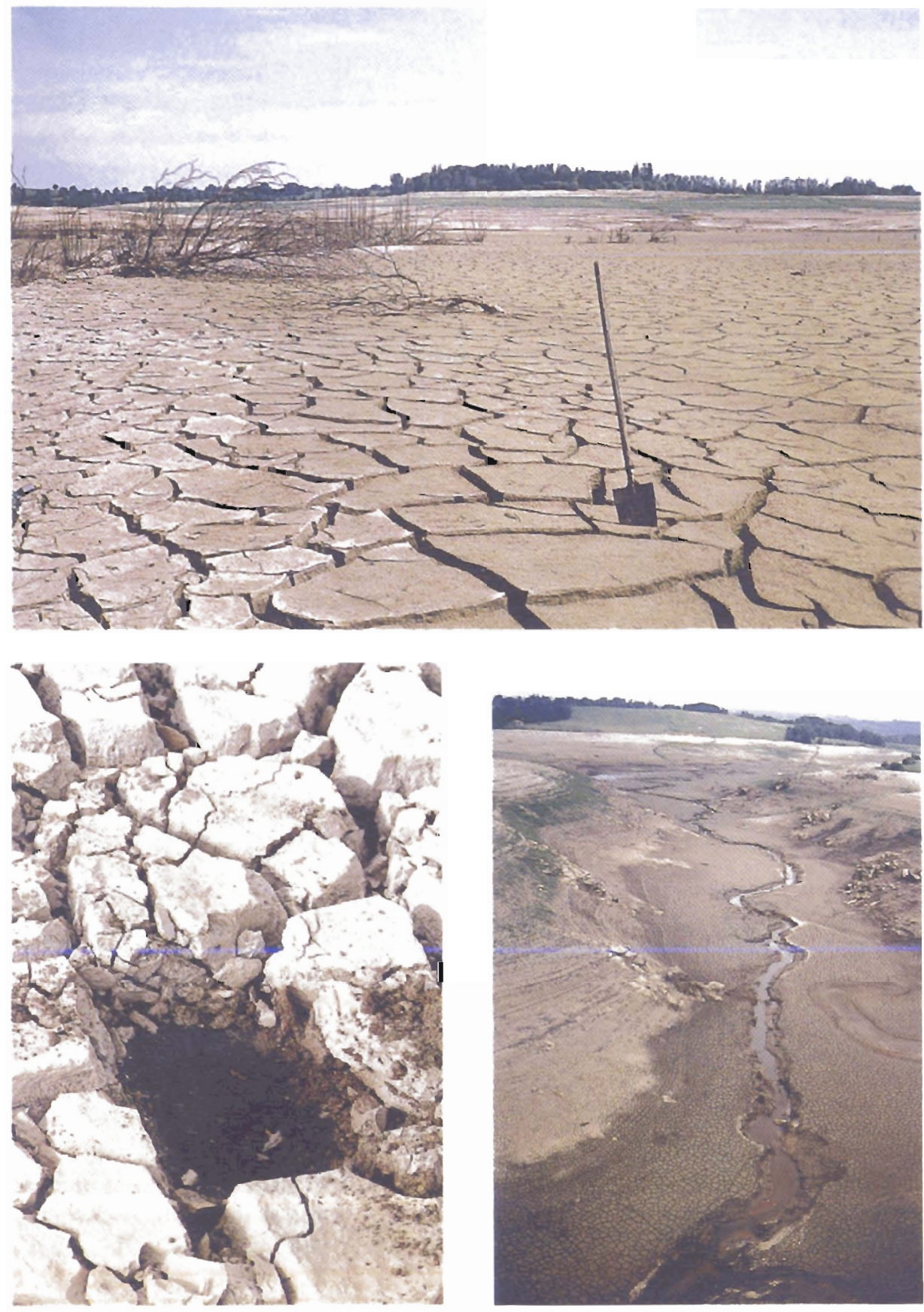

Planche II.

Plate II.

Photo 4. - Figures polygonales et fentes de retrait, dans la plaine principale aux abords du Charouzech.

Photo 4. - Polygonal figures and contraction gaps in the main valley near Le Charouzech.

Photo 5. - Quarante ans de dépôt sédimentaire sur un ancien sol agricole de la plaine du Vioulou.

Photo 5. - Forty years of sedimentary deposits on former agricultural land in the Vioulou plain.

Photo 6. - Dépôts en voie d'assëchement dans le vallon du Rieutord.

Photo 6. Drying deposits in the Rieutord valley. 
lées. En fait depuis quarante ans ces dépôts fins sont venus se superposer à l'ancienne terrasse alluviale des principaux cours d'eau contribuant à l'alimentation du réservoir. On peut évaluer cette surface à $4,5 \mathrm{~km}^{2}$, le reste de la cuvette étant occupé par des sables, de granulométrie variable en fonction de la structure de la roche dont ils sont issus, de graviers, cailloux, galets et rochers.

Si l'on veut estimer la quantité de dépôts fins produite depuis la mise en eau du réservoir, c'est à ces $4,5 \mathrm{~km}^{2}$ qu'il faut la rapporter. Sachant qu'un décimètre cube de sédiment fin séché à l'étuve à $105^{\circ} \mathrm{C}$ pendant 48 heures pèse $960 \mathrm{~g}$ (soit $960 \mathrm{~kg}$ par $\mathrm{m}^{3}$ de sédiment) et prenant comme épaisseur moyenne des dépôts $15 \mathrm{~cm}$, nous pouvons proposer une valeur de 648000 tonnes pour la totalité des limons déposés pendant 42 ans depuis la mise en eau, soit un rythme de sédimentation de $3429 \mathrm{t} \cdot \mathrm{km}^{-2} \cdot \mathrm{an}^{-1}$.

\section{PRINCIPAUX CARACTĖRES PHYSICO-CHIMIQUES DES SÉDIMENTS}

\section{IV.1 Quelques paramètres physico-chimiques}

Les dépôts fins ont une densité proche de 2,40 qui reflète assez bien la matrice minéralogique des principaux composants (opale $=2,1$, micas $=2,8$ ) et évoque la densité moyenne de la Montmorillonite.
Le pH du sédiment en place et encore sous l'eau présente des valeurs proches de la neutralité, surtout dans les premiers centimètres, mais séché à l'air, et mesuré selon la méthode standard appliquée au sol, il est acide et voisin en moyenne de 5,2 (4,80 pour les mêmes échantillons séchés à l'étuve à $105^{\circ} \mathrm{C}$ ). Le potentiel d'oxydo-réduction (Eh) mesuré à différentes profondeurs du sédiment nous renseigne sur l'état d'oxydation des horizons successifs. Dans les sédiments lacustres acides, comme c'est le cas ici, c'est le fer qui joue le rôle principal d'échangeur d'électrons (mais aussi le manganèse, les oxydes d'azote et le soufre). Après la vidange on a pu constater sur de grandes étendues le phénomène d'oxydation du fer à la surface des dépôts exondés (photo $n^{\circ} 8$ ). C'est dans la totalité de son épaisseur que le sédiment a vu son Eh augmenter au fur et à mesure de son aération.

Des mesures de Eh réalisées en laboratoire sur des sédiments, conservés dans leur état d'humidité maximale et immergés dans la même eau qu'à l'instant du prélèvement, montrent des variations de $600 \mathrm{mV}$ à $350 \mathrm{mV}$ de la surface vers les deux premiers centimètres du sédiment pour un $\mathrm{pH}$ voisin de 6 . et en valeurs corrigées par rapport à l'électrode à hydrogène.

Une fois séché, le sédiment a beaucoup de mal à se réhumecter; un bloc séché à l'air et immergé dans un récipient contenant de l'eau du Vioulou est resté intact pendant six mois, sa structure n'ayant pratiquement pas 

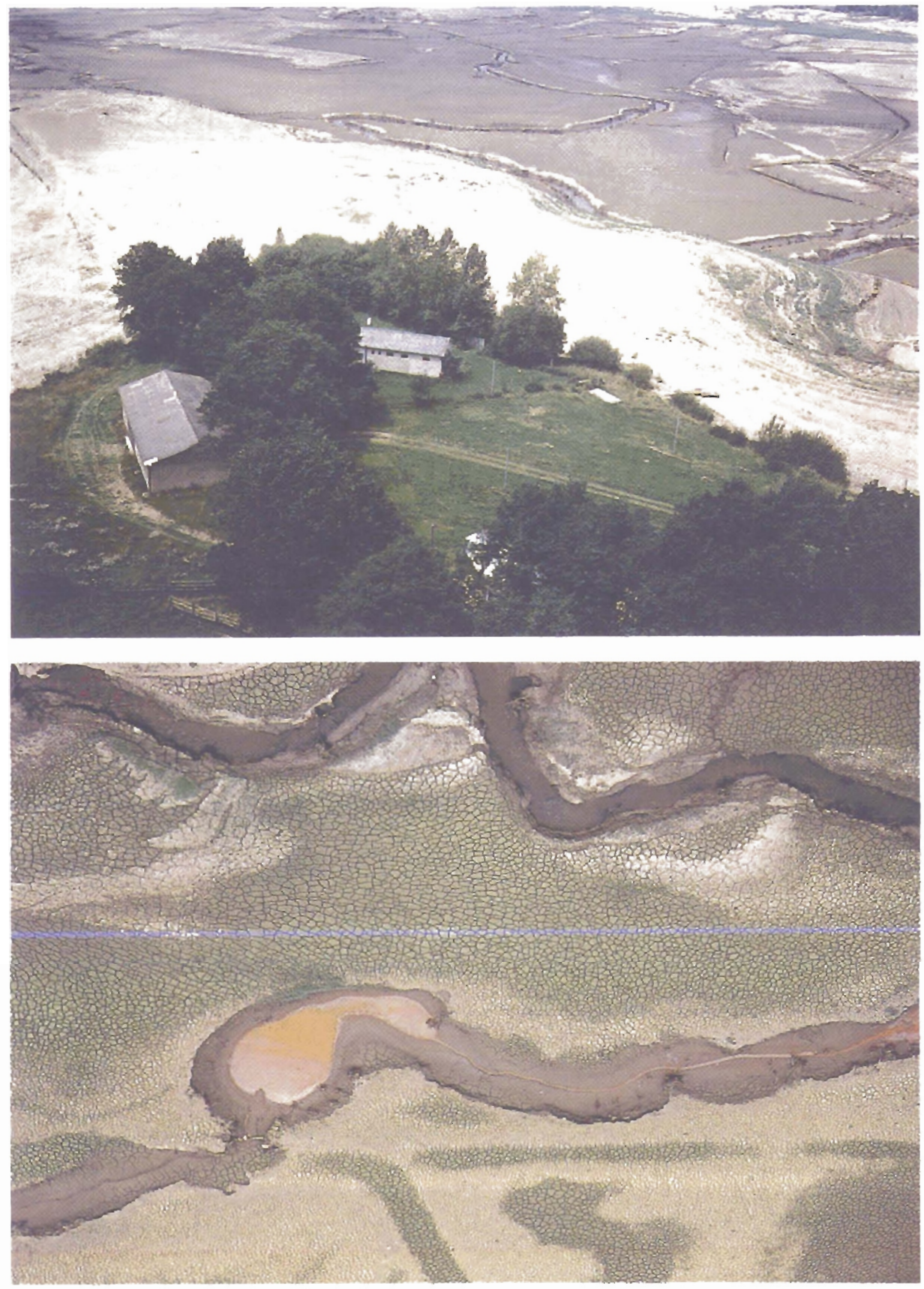

Planche III / Plate III

Photo 7. - Le laboratoire EDF à l'ouest de la presqu'ile du Charouzech, promontoire circonscrit par une plage de sables et graviers; au fond la plaine principale avec les méandres du Vioulou. Photo 7. - The EDF laboratory west of the Charouzech peninsula, a promontory surrounded by sand and gravel beach; in the background, the main plain with the Vioulou.

Photo 8. - Image montrant une plage de précipitation d'oxydes de fer lors du retrait des eaux dans un bras mort du Vioulou.

Photo 8. - Stretch of iron oxide deposits uncovered as the water recedes in an oxbow of the Vioulou. 
été altérée. Ce phénomène est dû à la compaction du sédiment lors de l'assèchement en présence de matière organique. Cette dernière, microparticulaire et bien répartie dans la masse du sédiment, devient hydrophobe en séchant et empêche le limon, qui présente par ailleurs une faible porosité, de se réhumecter par capillarité.

Pendant la période d'essai de mouillage le Eh n'a pas changé, il est resté voisin de $450 \mathrm{mV}$ dans le premier centimètre à la surface du bloc, les composés ferriques étant maintenus dans le même état d'oxydation. S'il en est de même dans le lac lors de la remise en eau, on doit s'attendre à des échanges limités entre le sédiment et l'eau pendant les premiers mois de remplissage, notamment par rapport au phosphore $\left(\mathrm{PO}_{4}^{3-}\right)$ lié au fer $\left(\mathrm{Fe}^{3+}\right)$ qui se trouve ainsi immobilisé dans le sédiment.

\section{IV.2 Granulométrie de la fraction minérale}

La texture a été déterminée sur 39 échantillons prélevés à la benne Eckmann en 1987 et sur 26 échantillons prélevés "de visu " à la bêche après le retrait des eaux lors de la vidange de 1993. Pendant la période de baisse progressive du niveau d'eau, deux séries de prélèvements ont été réalisés, l'une en mars/avril 1993 (série P) portant sur 14 échantillons, l'autre en juillet 1993 (série J) de 12 échantillons concernant essentiellement la cuvette principale.
La série de 1987 et celle de juillet 1993 ont été analysées à l'aide d'un granulomètre laser type CILAS ALCATEL M.715, celle du printemps 1993 n'ayant pu être traitées que par la méthode classique à la pipette de Robinson (fig. 3).

De l'analyse des résultats il se dégage quelques constatations essentielles:

- les sédiments de la cuvette principale présentent une grande identité de texture et les fractions inférieure à $50 \mu \mathrm{m}$ dominent largement, l'ensemble [limons grossiers + limons fins + argiles] étant toujours supérieur à $80 \%$ et pouvant même atteindre $90 \%$;

- les argiles proprement dites ne dépassent que très rarement des taux de $20 \%$ (en fait $10 \%$ pour les échantillons traités au granulomètre laser), ce sont donc les limons qui dominent largement partout dans les fractions inférieures à $50 \mu \mathrm{m}$;

- au débouché des principaux ruisseaux, l'hétérogénéité spatiale est plus grande (fig. 4) et on peut aller de la texture sableuse à la texture limoneuse, ceci étant bien sûr lié à la configuration géomorphologique du site d'échantillonnage et à la distance du confluent au lieu de prélèvement;

- en moyenne les sédiments à l'embouchure du Connes montrent une dominance des limons fins et des sables fins ;

- pour le talweg du Vioulou on notera une nette dominance des limons fins et une distribution presque identique des limons grossiers et des sables fins ; 

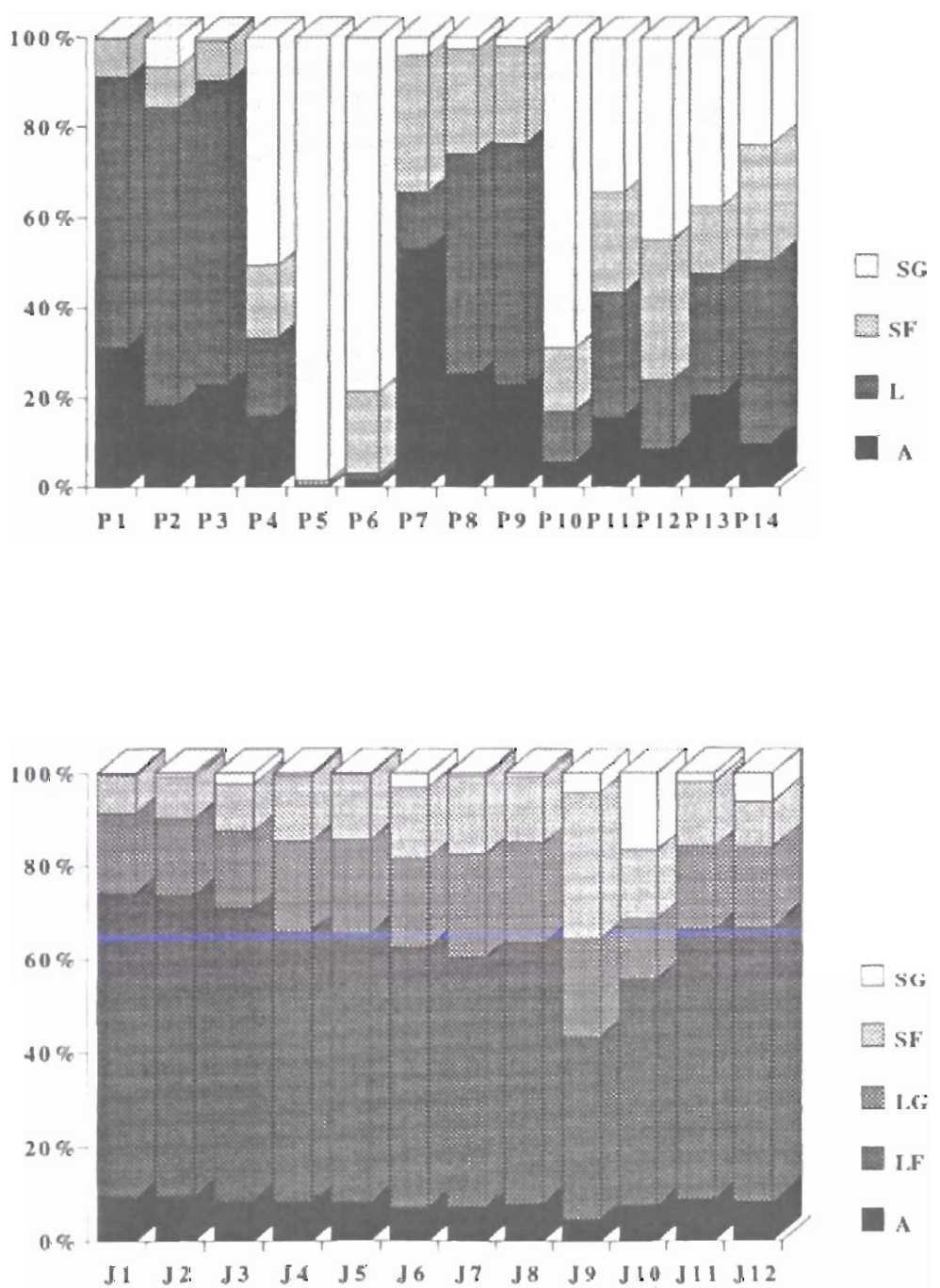

Fig. 3. - Texture des sediments prélevés en mars-avril (P) et juillet (J) 1993.

Fig. 3. - Textural composition of sediments sampled in March-April (P) and July (J) 1993. 
CONNES

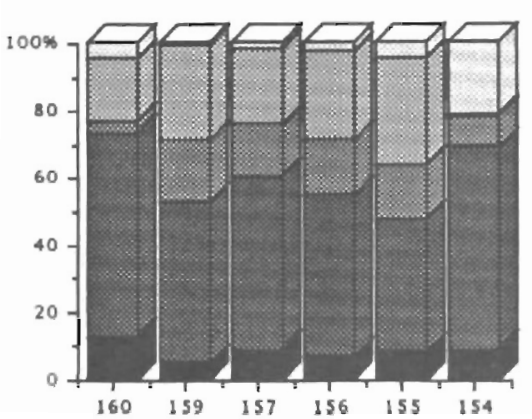

MAS ATCHE

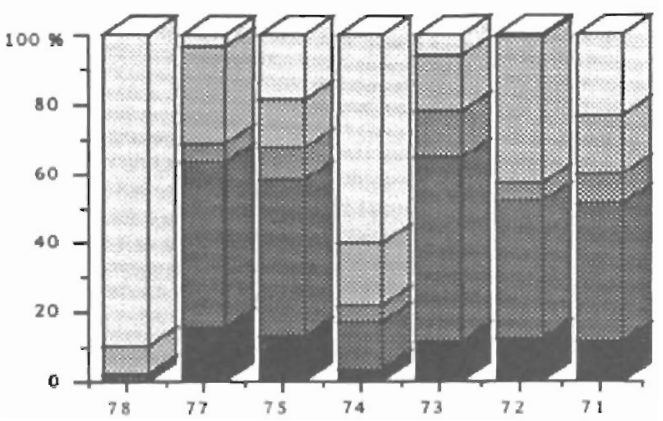

VIOULOU

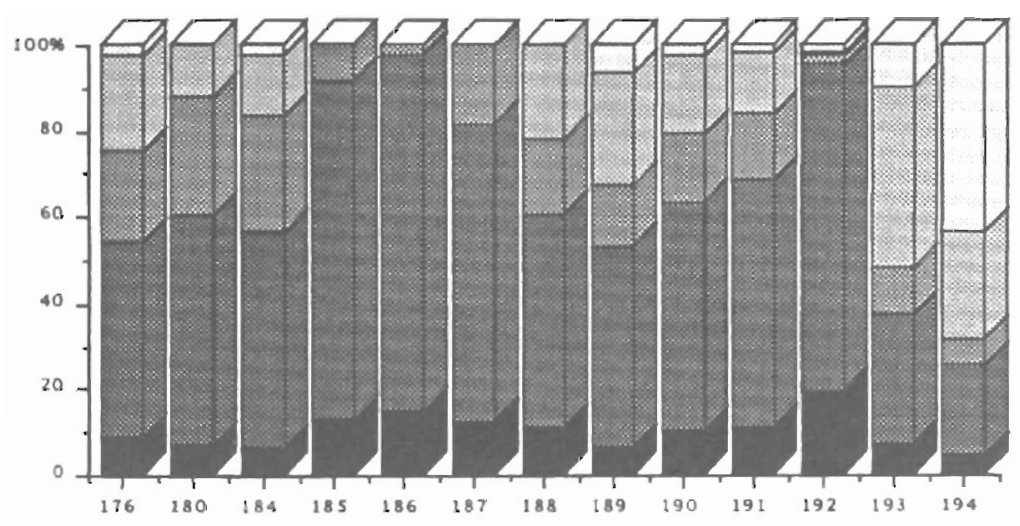

RIEUTORD

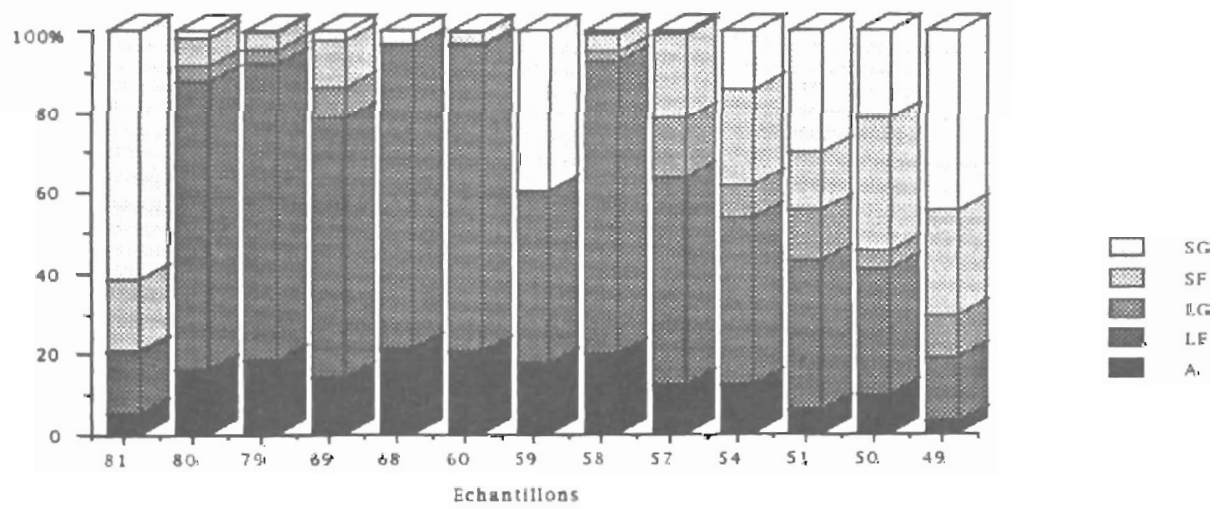

Fig. 4. - Texture des sédiments dans les quatre anses principales du lac de Pareloup.

Fig. 4. - Textural composition of sediments in the four principal coves in Pareloup reservoir. 
- pour celui du Mas Atché une légère diminution des taux des limons au profit des sables;

- pour le vallon du Rieutord une moins bonne représentation des limons grossiers au profit des sables grossiers.

\section{IV.3 Les éléments minéraux essentiels}

Les tableaux III et IV présentent les teneurs en éléments minéraux totaux provenant des analyses des échantillons des campagnes successives réalisées en 1986 et 1993.

La silice provenant de la dissolution des roches du bassin versant, mais aussi et surtout de la remobilisation par les diatomées, et alors sous forme d'opale, est présente à raison de $33,4 \%$ à $40 \%$ et l'aluminium à raison de $11 \%$ à $11,9 \%$ en moyenne.

Les autres minéraux se rencontrent avec des taux beaucoup plus faibles et dans l'ordre décroissant on peut noter : le fer de 38,4 à $44,1 \%$ assez

Tableau III. - Éléments chimiques totaux des sédiments au débouché des principaux ruisseaux (1986).

Table III. - Total chemical elements in sediments at the outlet of the principal streams (1986).

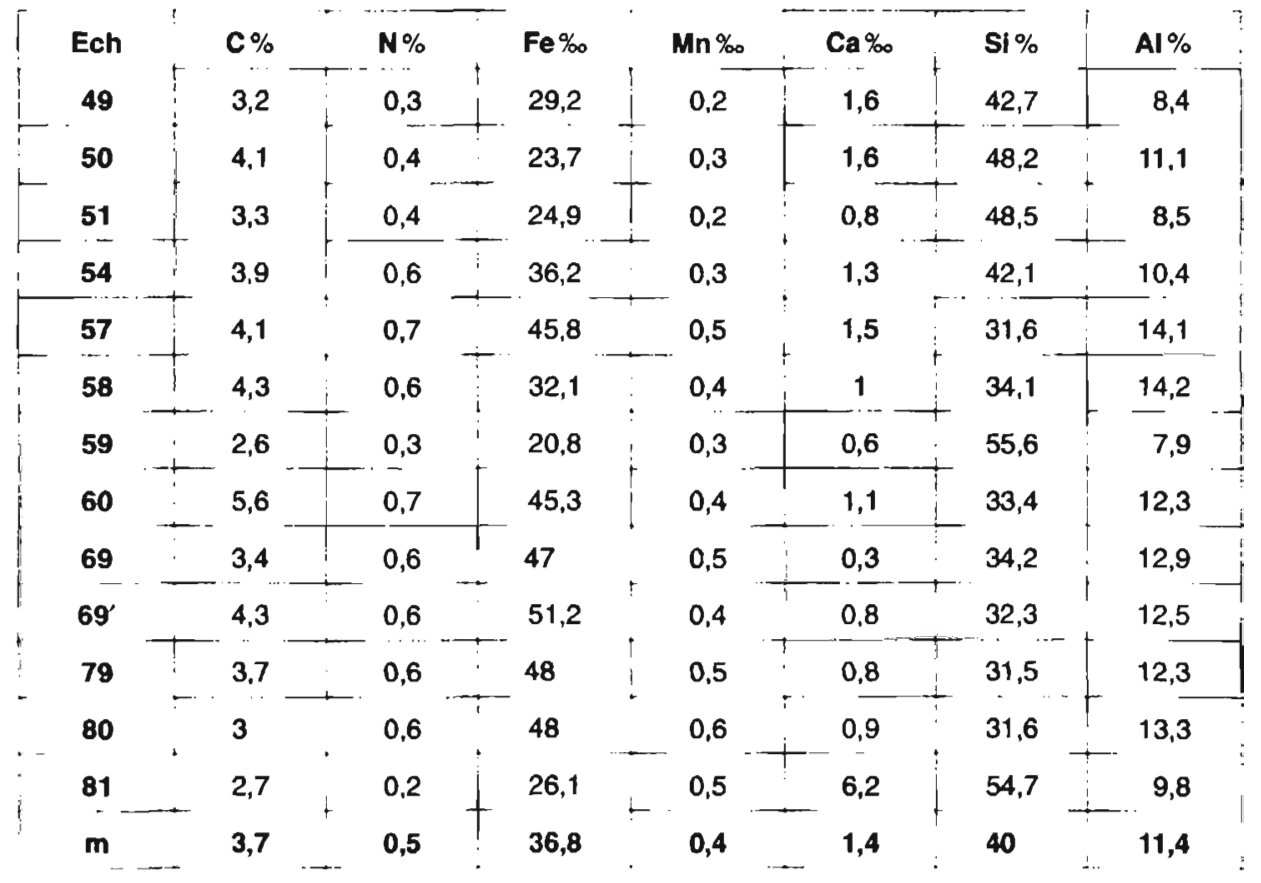

\section{RIEUTORD}




\begin{tabular}{|c|c|c|c|c|c|c|c|}
\hline Ech & C \% & $\mathbf{N} \%$ & $\mathrm{Fe} \%$ & Mn \% & $\mathrm{Ca} \%$ & Si \% & Al $\%$ \\
\hline 71 & 3,8 & 0,4 & 30,3 & 0,4 & 0,6 & 43,9 & 10,3 \\
\hline 72 & 3,5 & 0,5 & 60,5 & 0,3 & 0,7 & 29,8 & 12,7 \\
\hline 73 & 4,2 & 0,5 & 37 & 0,4 & 0,8 & 37,2 & 12,1 \\
\hline 75 & 2,5 & 0,4 & 45,1 & 0,4 & 0,6 & 40,4 & 11,1 \\
\hline$\pi$ & 3,7 & 0,5 & 47,6 & 0,5 & 1,5 & 28,2 & 13 \\
\hline $\mathbf{m}$ & 3,5 & 0,46 & 44,1 & 0,4 & 0,8 & 35,9 & 11,8 \\
\hline
\end{tabular}

MAS ATCHÉ

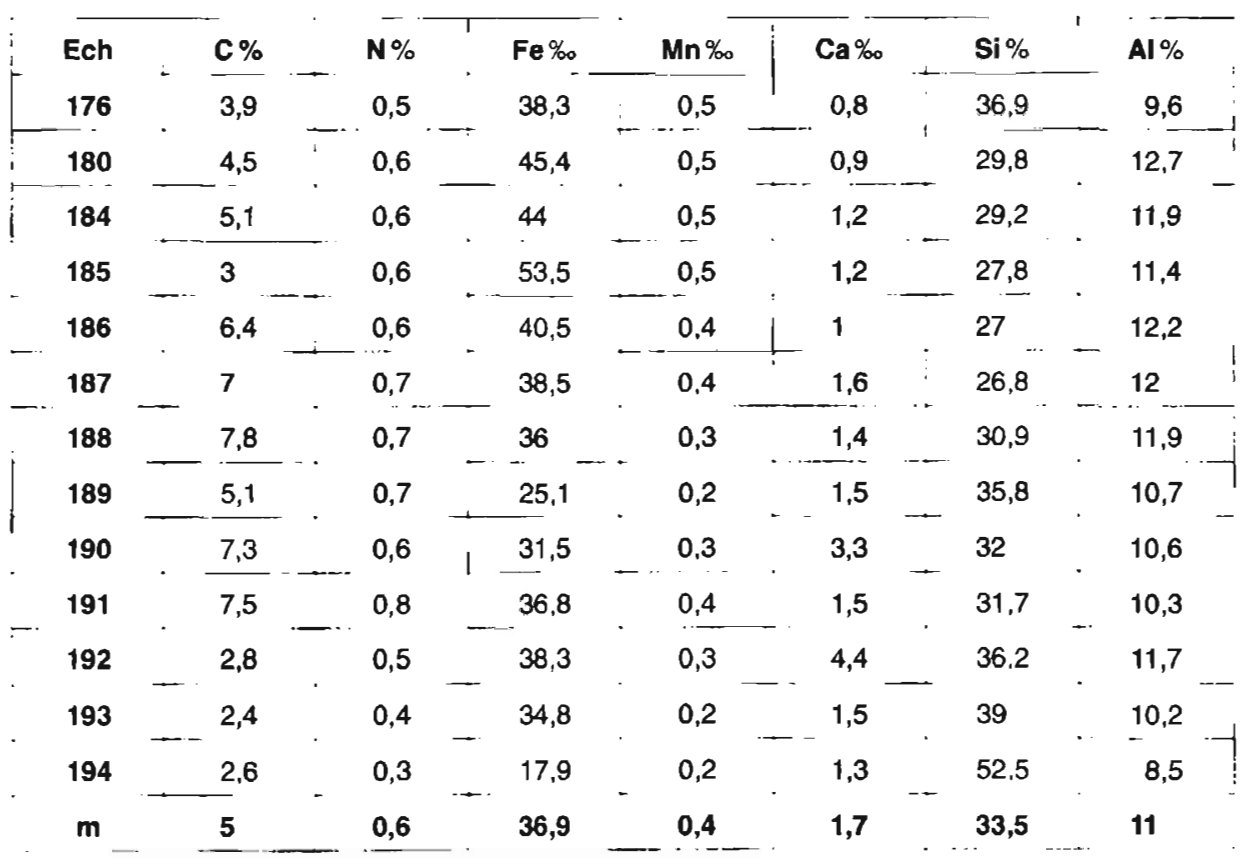

VIOULOU

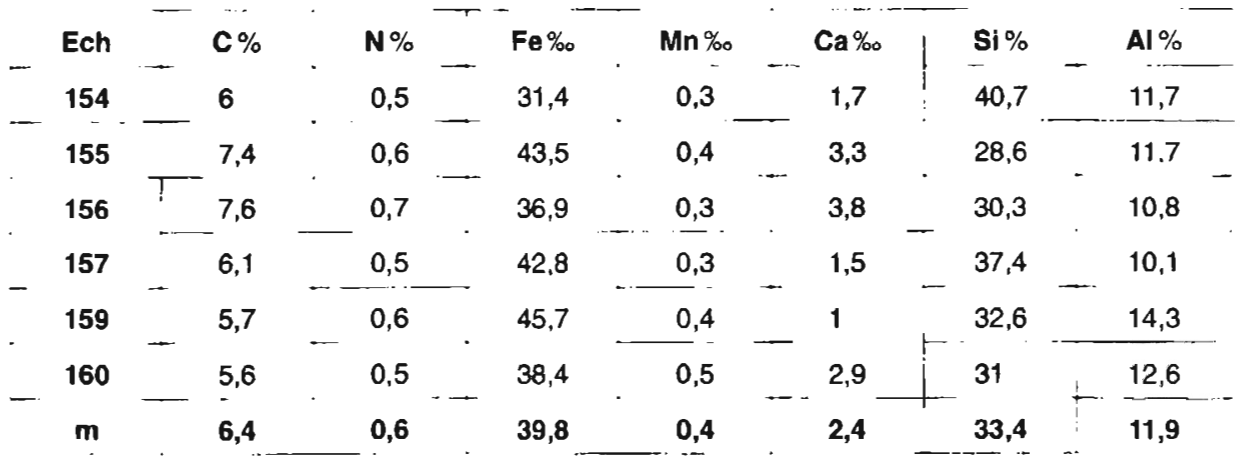


Tableau IV. - Teneurs en éléments chimiques totaux des sédiments prélevés lors de la vidange de 1993.

Table IV. - Total chemical concentrations in sediments sampled during the 1993 draining.

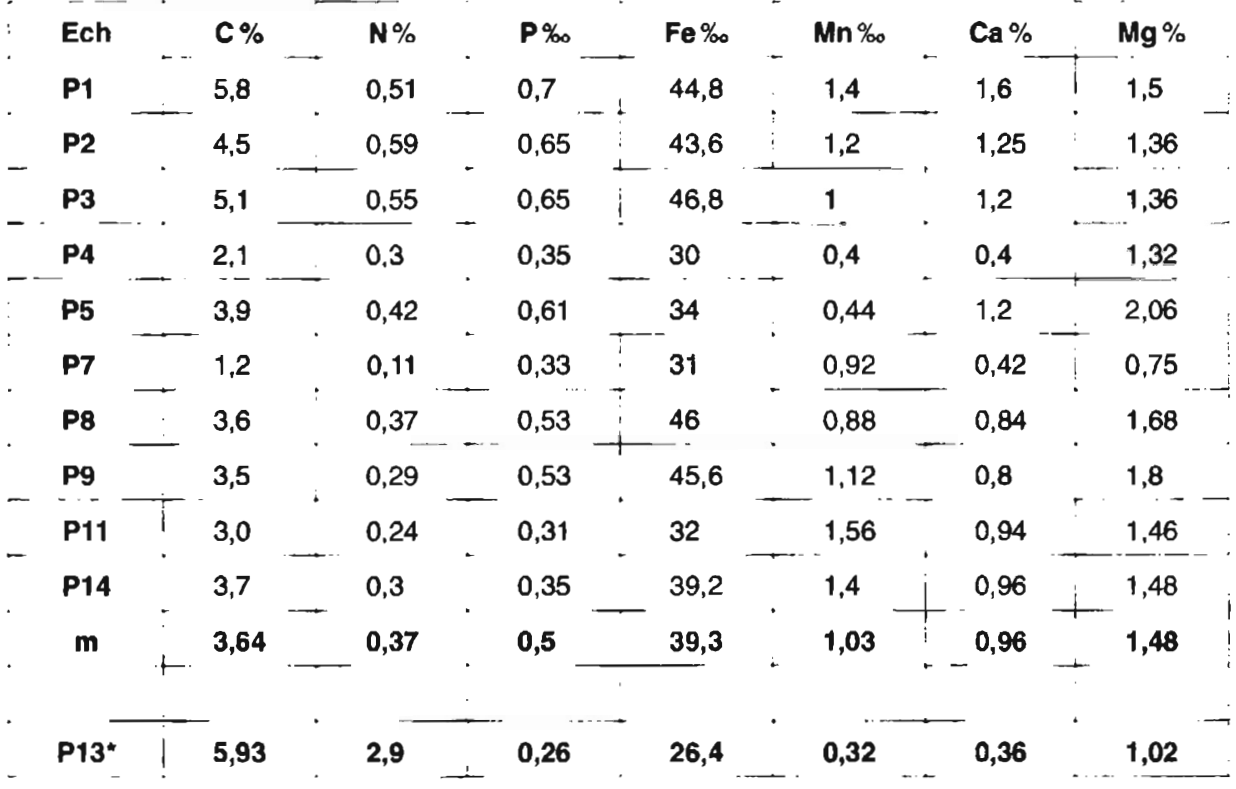

* Ancien sol de lande à genêts.

\begin{tabular}{|c|c|c|c|c|c|c|c|c|c|}
\hline Ech & C \% & $\mathbf{N} \%$ & 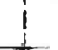 & P\% & $\mathbf{F e} \%$ & $M n \%$ & Ca $\%$ & & $\mathbf{M g} \%$ \\
\hline J1 & 4,7 & 0,51 & | & 1,1 & 42,6 & 1 & 0,62 & & 1,9 \\
\hline J2 & 4,5 & 0,53 & & 1,1 & 42,2 & 1,04 & 0,4 & & 1,84 \\
\hline J3 & 4,4 & 0,54 & i & 1,1 & 44,8 & 1,14 & 0,34 & 1 & 1,72 \\
\hline J4 & 5,4 & 0,55 & 1 & 1 & 42 & 1,06 & 0,56 & & 2,24 \\
\hline J5 & 5,7 & 0,57 & ! & 1 & 42 & 1 & 0,61 & & 2,24 \\
\hline J6 & 6 & 0,68 & $\therefore$ & 1 & 42,8 & 1,26 & 0,8 & & 2,4 \\
\hline J7 & 5,4 & 0,54 & 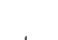 & 1,1 & 38 & 0,82 & 0,4 & & 1,82 \\
\hline J8 & 5 & 0,51 & & 1,1 & 38,8 & 0,92 & 0,54 & & 1,84 \\
\hline J9 & 4,5 & 0,43 & ' & 0,95 & 30,4 & 0,76 & 0,52 & 1 & 2,66 \\
\hline J10 & 3,8 & 0,41 & & 0,85 & 28,2 & 0,59 & 0,38 & & 1,24 \\
\hline J11 & 6,7 & 0,63 & & 1 & 35,6 & 0,9 & 0,65 & & 1,68 \\
\hline $\mathbf{J 1 2}$ & 5,3 & 0,5 & & 1 & 33,2 & 0,96 & 0,64 & & 1,58 \\
\hline $\mathbf{m}$ & 5,1 & 0,53 & & 1,03 & 38,4 & 0,95 & 0,54 & & 1,93 \\
\hline
\end{tabular}


abondant, le magnésium de 1,48 à $1,93 \%$, le calcium de 0,54 à $2,4 \%$, le manganèse de 0,4 à $1,03 \%$.

Une mention particulière peut être faite pour le phosphore vu son rôle important dans le cycle biogéochimique. La dissolution des roches permet sa mise en solution et son transport sous forme de particules en suspension.

Dans les roches éruptives, l'abondance du phosphore est, d'après Vanwazer (1961) (in T. Ahl 1988), de 0,10 à $0,12 \%$ de $P$. Ceci est très voisin des valeurs trouvées dans les sédiments de Pareloup.

Par rapport aux valeurs annoncées dans la littérature existante sur le sujet, les valeurs que nous obtenons sont, pour un environnement de sols acides, d'un ordre de grandeur convenable puisqu'elles varient de $0,5 \%$ à $1,03 \%$. Le taux de $3 \%$ ost, dans des conditions assez voisines d'environnement, celui proposé par A. Fabre [8] lors de son étude du réservoir de Puyvalador (P.O.). Les résultats de nos analyses sont assez voisins de ceux proposés par G. Rofes [16] lors des premiers prélèvements de sédiment à Pareloup en 1983.

La géologie, la géomorphologie, la morphométrie ef l'occupation des sols sont des facteurs importants pour ce qui concerne le cycle du phosphore. La surface couverte par les forêts, la surface des marges, les zones marécageuses et le temps de rétention de l'eau dans le lac influencent fortement les concentrations en phosphore du sédiment.

\section{IV.4 La matière organique}

La matière organique des sédiments lacustres a une triple origine; elle provient :

- des matières en suspension et des matières dissoutes (COD) transportées par les ruisseaux ayant drainé les différents bassins versants;

- des composés organiques arrachés par l'érosion aux talus et berges du plan d'eau lui-même ;

- des différents organismes vivants dans la masse d'eau et de leurs excrétions ou de leurs restes.

Une partie importante de la matière organique produite dans le lac est recyclée rapidement dans la colonne d'eau et reprise dans la chaine alimentaire. Celle rencontrée dans les sédiments correspond à la fraction résiduelle. Dans les sédiments fins de la cuvette principale, qui forment ici la plus grande partie des dépôts comme nous l'avons souligné, la matière organique se trouve sous forme de fines particules, et du fait que les abords du plan d'eau sont très peu boisés dans l'ensemble on ne trouve que très peu de matériel végétal grossier et surtout aucune accumulation de feuilles ou de débris végétaux grossiers comme dans d'autres réservoirs entourés de forêts.

Les teneurs en matière organique des sédiments sont à Pareloup comprises entre $6 \%$ et $11,5 \%$, avec 
une moyenne de $7,5 \%$ (ces taux sont calculés à partir du carbone organique dosé par voie chimique). Sur les talus ou dans les anses (Rieutord, Mas Atché, Vioulou) les sédiments ont une texture nettement plus variable, le matériel minéral est plus hétérogène et les taux de matiere organique beaucoup plus variables. Pour le vallon de Rieutord et pour quatorze échantillons prélevés à la benne Eckmann en 1986, la teneur moyenne en matière organique est de $6,4 \%$ et de $8,6 \%$ pour treize échantillons provenant de l'anse au débouché du Vioulou. Pour l'anse du Connes on note une valeur moyenne de $11 \%$. Les valeurs extrêmes sont cependant pour cette campagne de $2,1 \%$ pour le minimum et $13,4 \%$ pour le maximum. Les teneurs en azote lié à la matière organique sont en moyenne de $0,37 \%$ à $0,6 \%$ avec des écarts à la moyenne relativement faibles. Les rapports $\mathrm{C} / \mathrm{N}$, qui donnent une bonne idée du taux de minéralisation et de la nature probable des composés organiques, sont ici généralement inférieurs à 10 , la valeur moyenne étant de 9,6 pour les dépôts fins de la plaine principale et variant de 7,4 à 10,6 pour les dépôts des principaux bras latéraux. Les valeurs extrêmes du rapport $\mathrm{C} / \mathrm{N}$ sont de 5 et 12,5. La valeur exceptionnelle de 20 trouvée en P13 correspond en fait à un ancien sol exondé de type ranker s'étant développé sous une lande à genêt avant la mise en eau du barrage et ayant gardé ses caractéristiques pédologiques originelles (humus de type moder). Dans les lacs assez profonds et a forte production primaire du Jura, les rapports $\mathrm{C} / \mathrm{N}$ demeurent bas (voisins de 8).

Hansen (1959) donne le nom de gyttja à un sédiment dont la matière organique est voisine de $15 \%$ et le rapport $\mathrm{C} / \mathrm{N}$ inférieur à 10 , c'est le cas ici.

Nous pouvons constater au vu du tableau $V$ qu'ici ces deux paramètres évoluent très peu dans l'épaisseur du sédiment, donc avec le temps, ceci semble traduire un certain équilibre dans les conditions de milieu depuis au moins dix ans.

L'histoire que peut renfermer un sédiment dépend tout autant des caractéristiques physiques, chimiques et biologiques du bassin versant, que de celles afférent à la masse d'eau ellemême. Le taux de matière organique dans le sédiment, étant exprimé en pour cent du poids de sédiment, dépend donc de la fluctuation dans la production de matière organique dans la masse d'eau, des apports de cette dernière par les différents vecteurs, ainsi que de la fluctuation de la vitesse d'érosion du compartiment minéral du bassin versant et des berges.

La question fondamentale reste donc posée de pouvoir déterminer dans le stock de matière organique du sédiment la part relative des différentes sources et leur degré d'évolution. Ceci rejoint l'affirmation de J. Verneaux et al. [20] dans les conclusions de leur article sur la MO des lacs du Jura: "La nature de la $\mathrm{MO}$ résiduelle influence donc considérablement la valeur du rapport $\mathrm{C} / \mathrm{N}$ 
Tableau V. - Variations des teneurs en éléments chimiques totaux dans les cinq premiers centimètres du sédiment.

Table V. - Variation in total chemical concentrations in the first five centimeters of sediment.

\begin{tabular}{|c|c|c|c|c|c|c|c|c|}
\hline Ech & C \% & $\mathbf{N} \%$ & $C / N$ & $\mathbf{P} \%$ & $\mathrm{Fe} \%$ & Mn \% & $\mathrm{Ca} \%$ & $\mathbf{M g} \%$ \\
\hline $0-0,5$ & 5,00 & 0,45 & 11,10 & 1,00 & 44,00 & 1,16 & 1,20 & 2,20 \\
\hline $0,5-1$ & 5,30 & 0,49 & 10,80 & 0,95 & 42,20 & 1,08 & 0,64 & 1,92 \\
\hline $1-1,5$ & 4,70 & 0,47 & 10,00 & 1,00 & 44,60 & 1,12 & 0,62 & 2,20 \\
\hline $1,5-2$ & 4,40 & 0,50 & 8,80 & 1,00 & 42,20 & 0,90 & 0,56 & 2,10 \\
\hline $2-2,5$ & 4,50 & 0,49 & 9,20 & 0,83 & 34,00 & 0,66 & 0,54 & 1,80 \\
\hline $2,5-3$ & 4,40 & 0,47 & 9.40 & 0,87 & 40,00 & 0,78 & 0,76 & 2,58 \\
\hline $3-3,5$ & 4,30 & 0,46 & 9,30 & 0,99 & 44,80 & 0,72 & 0,52 & 2,50 \\
\hline $3,5-4$ & 4,40 & 0,47 & 9,40 & 0,99 & 41,20 & 0,70 & 0,52 & 2,20 \\
\hline $4-4,5$ & 4,20 & 0,47 & 8,90 & 0,87 & 36,80 & 0,62 & 0,46 & 2,10 \\
\hline $4,5-5$ & 4,70 & 0,49 & 9,60 & 0,74 & 34,00 & 0,56 & 0,40 & 1,84 \\
\hline$>5$ & 4,20 & 0,50 & 8,40 & 0,78 & 35,20 & 0,98 & 0,34 & 1,96 \\
\hline Moyenne & 4,60 & 0,48 & 9,50 & 0,89 & 39,90 & 0,84 & 0,60 & 2,13 \\
\hline
\end{tabular}

indépendamment de la teneur en MO».

\section{CONCLUSIONS}

La vidange du réservoir de Pareloup nous a permis de comparer les diverses méthodes d'évaluation du taux de sédimentation et d'estimer avec une certaine précision le rythme annuel des dépôts; chaque année une pellicule de $0,5 \mathrm{~cm}$ de sédiment (épaisseur rapportée au sédiment séché à l'air) vient s'ajouter aux dépôts déjà consolidés et correspond à un poids de $3429 \mathrm{t} \cdot \mathrm{km}^{-2}$. an ${ }^{-1}$ de sédiment sec. Cette quantité est plus de dix fois supérieure à celle calculée à partir des seuls apports de MES des trois principaux ruisseaux alimentant le plan d'eau $\left(232 \mathrm{t} \cdot \mathrm{km}^{-2} \cdot \mathrm{an}^{-1}\right)$ et deux fois plus importante que celle estimée à partir des résultats obtenus avec les pièges à sédiments. La valeur bien trop faible obtenue par la seule addition des apports annuels de MES des ruisseaux se justifie par le fait que manquent les estimations des apports dus à la production primaire du lac et ceux résultant de l'érosion des berges et du bassin versant direct $\left(40 \mathrm{~km}^{2}\right)$ qui représentent certainement des quantités fort importantes et qu'il faudrait pouvoir chiffrer.

Les résultats obtenus à partir des pièges à sédiments posés pendant 
4 saisons successives sont ici plus proches de la réalité, mais cette méthode d'estimation dépend fortement de la géomorphologie du bassin. Les sédiments déposés sur le talus sont, dès que la pente devient un peu forte, repris par l'action des vagues, des courants ou de la gravité et vont rejoindre ceux accumulés sur le fond du bassin après une période de remise en suspension plus ou moins longue suivant leur taille et leur densité.

Les valeurs obtenues pour le lac de Pareloup sont tout à fait plausibles eu égard au contexte géomorphologique et climatique si on es compare à ceux avancés par les différents auteurs ayant traité des mêmes problèmes.

Le fond de la cuvette principale est composé d'un sédiment limoneux assez homogène, d'une épaisseur moyenne de 15 centimètres, refermant près de $10 \%$ de matière organique finement particulaire et peu humifiée.

Dans les anses, les dépôts sont variés du point de vue de leur texture et de leur distribution géographique. Les dépôts sont riches en fer et ce dernier joue un rôle essentiel dans les échanges eau-sédiment; cette abondance en fer, généralement typique des régions cristallines, est du au fait que les sols, souvent comme ici de type ranker, cedent par percolation et ruissellement des eaux d'importantes quantités de cet élément lié aux complexes organiques qui les caractérisent.

Du fait des très faibles teneurs en carbonate de calcium, c'est le fer qui joue un rôle essentiel lors des échanges de phosphore entre le sédiment et l'eau.

En dehors de la silice et de l'aluminium $(40 \%$ et $11 \%)$, les autres éléments sont moyennement représentés comme le manganèse et le phosphore ( $1 \%$ ) ou faiblement présents comme le calcium et le magnésium $(0,5$ et $2 \%)$.

La vidange de la retenue, qui a laissé le bassin à sec pendant l'été 1993, permettra aussi, grâce à la réalisation de photographies aériennes, de proposer prochainement une cartographie détaillées des sédiments exondés. Cette cartographie n'avait pu être tentée en 1987 par une méthode moins aisée alors que le réservoir était en eau, par suite de conditions météorologiques défavorables; cependant les présents résultats confirment dans l'ensemble ceux obtenus par les carottages réalisés cette année-là.

Les contraintes rencontrées aujourd'hui pour la gestion de la sécurité des retenues rendent nécessaire la mise au point de techniques physiques (acoustique, géophysique) qui permettront d'estimer la qualité et la quantité des sédiments déposés dans les réservoirs avant vidange.

Le Laboratoire d'Hydrobiologie en association avec d'autres laboratoires (LAMI Toulouse, DTG Grenoble) tente une telle démarche. La présente étude pourrait d'ailleurs servir de référence pour l'étalonnage des mesures in situ. 


\section{BIBLIOGRAPHIE}

[1] Ahl T., 1988. Background yeld of phosphorus from drainage area and atmosphere: An empirical approach. Hydrobiologia, $170: 35-44$.

[2] Bosc Ph., 1985. Evaluation des apports en éléments nutritifs du bassin versant dans le lac de Parloup (Aveyron). Dea Univ. Toulouse III : 123 pp.

[3] Bücher A. \& Lucas C., 1984. Sédimentation éolienne intercontinentale, poussières sahariennes et géologie. Bull. Centres Rech. Explor.-Prod. ElfAquitaine, Pau, 8(1) : 151-165.

[4] Butcher D.P., Labadz J.C., Potler A.W.R. and white P., 1993. Reservoir sedimentation rates in the southern Pennine region, Uk. Geomorphology and sedimentology of Lakes and Reservoirs. Edited by J. McManus and R.W. Duck. John Wiley \& Sons Ltd: 73-92.

[5] Dearing J.A. and Foster I.D.L., 1993. Lake sediments and geomorphological Processes : some thoughts. Geomorphology and Sedimentology of Lakes and Reservoirs. Edited by $\mathrm{J}$. McManus and R.W. Duck. John Wiley \& Sons Ltd: 5-14.

[6] Dillon P.J., Evans R.D. and Molot L.A., 1990. Retention and resuspension of phosphorus, nitrogen, and iron in a Central Ontario lake. Can. J. Fish. Aquat. Sci., 47 : 1269-1274.

[7] Dussart B., 1991. Limnologie. Boubée ed., Paris : 681 pp.

[8] Fabre A. \& Patau-Albertini M.F., 1986. Sediment heterogeneity in a reservoir subject to heavy drawdown. Hydrobiologia, 137 : 89-94.

[9] Feuillade J. \& Feuillade M., 1971. Etude comparative en 1966 et 1967 , de trois lacs de retenue du projet de parc naturel régional du Morvan. Annls. Hydrobiol., 2 (2) : 143-174.

[10] Hakanson L., 1981. Determination of characteristic values for physical and chemical lake sediment parameters.
Water Resources Research, 17 (6) : 1625-1640.

[11] Hakanson L. \& Jansson M., 1983. Principles of lake sedimentology. Springer-Verlag ed., Berlin : 316 pp.

[12] Hansen K., 1959. The terms Gyttja and Dy. Hydrobiologia, 13 : 309-315.

[13] Hansen K., 1961. Lake types and lake sediments. Verh. Internat. Verein. Limnol., XIV : 285-290.

[14] Mackereth F.J.H., 1957. Chemical analysis in ecology illustrated from lake district tarns and lakes. I. Chemical analysis. Proc. Linn. Soc. Lond., 167 (2) : 159-164.

[15] Rhodes T.E. \& Le Cohu R., 1987. Sédiments et paléolimnologie d'un réservoir : le lac de Pareloup (Aveyron, france). Premiers résultats. Annals. Limnol., 23 (1) : 3-7.

[16] Rofes G., 1983. Etude de la retenue de Pareloup (Aveyron). Rapport ME/31-84.41, Electricité de France, Paris : $16 \mathrm{pp}$.

[17] Rofes G. et al., 1993. Dépôts et remises en suspension dans le lac de Grandlieu (France). Verh. Internat. Verein. Limnol., 25: 608-609.

[18] Salençon M.J., Thébault J.M. et Capblancq J., 1990. Étude de la retenue de Pareloup. Synthèse des travaux réalisés dans le cadre de la Convention EDF-Ministère de l'Environnement (mars 1986-mars 1990). Rapport HE-31/90.23-Électricité de France, Paris : $40 \mathrm{pp}$.

[19] Thébault J.M. and Salençon M.J., 1993. Simulation model of a mesotrophic reservoir (Lac de Pareloup) : biological model. Ecol. Modelling, 65: 1-30.

[20] Verneaux J., Vidonne A., Remy F. \& Guyard A., 1991. Particules organiques et rapport $\mathrm{C} / \mathrm{N}$ des sédiments des lacs du Jura. Annls. Limnol., 27 (2) : 175-190.

[21] Wetzel R.G., 1970. Recent and postglacial production rates of a marl lake. Limnol. Oceanogr., 15 : 491-503. 